\title{
- An Optimal Transaction Intervals for Portfolio Selection Problem with Bullet Transaction Cost
}

\author{
Effendi Syahril
}

\begin{abstract}
This paper discusses an optimal transaction interval for a consumption and investment decision problem for an individual who has available a riskless asset paying fixed interest rate and a risky asset driven by Brownian motion price fluctuations. The individual observes current wealth when making transactions, that transactions incur costs, and that decisions to transact can be made at any time based on all current information. The transactions costs is fixed for every transaction, regardless of amount transacted. In addition, the investor is charged a fixed fraction of total wealth as management fee. The investor's objective is to maximize the expected utility of consumption over a given horizon. The problem faced by the investor is formulated in a stochastic discrete-continuous-time control problem. An optimal transaction interval for the inverstor is derived.

Key words: Transaction intervals, investment strategy, bullet (fixed) transaction cost, continuous-discrete-time, stochastic optimal control problem
\end{abstract}




\section{Introduction}

The publication of Merton's seminal work, see Merton(1971), has started the application of stochastic optimal control and stochastic calculus techniques to the area of finance. Merton $(1971,1990)$ studied the behaviour of a single agent acting as a market price-taker who seeks to maximize expected utility of consumption. The utility function of the agent was assumed to be a power function, and the market was assumed to comprise a risk-free asset with constant rate of return and one or more stocks, each with constant mean rate of return and volatility. The only information available to the agent were current prices of the assets. There were no transaction costs. It was also assumed that the assets were divisible. In this idealized setting, Merton was able to derive a closed-form solution to the stochastic optimal control problem faced by the agent.

Several authors have made contributions to the stochastic optimal control and stochastic calculus analyses of the Merton's model. To mention a few among them are Constantinides (1979, 1986), Cox and Huang (1989), Davis and Norman (1990), Duffie and Sun (1990), Dumas and Luciano (1991), Lelands (1985), Magill and Constantinides (1976).

The application of transaction costs to Merton's model was first accomplished by Magill and Constantinides (1976). Several authors then have published a number of works on Merton's model with transaction costs. To mention a few, they are Constantinides (1979, 1986), Davis and Norman (1990), Duffie and Sun (1990), Dumas and Luciano (1991), Lelands (1985). Duffie and Sun (1990) treated the proportional transaction costs with different formulation to others, which they call discrete-continuous-time formulation. Their formulation assumes that an investor observes current wealth when making transaction, and decisions to transact can be made at any time, but without no costs. They treated general linear transaction costs of the form $a W_{\tau_{n}}+b$, with $W_{\tau_{n}}$ denotes the amount of wealth transacted, and $a$ and $b$ are non-negatives. Based on work of Duffie and Sun (1990), Syahril (2003a) re-writes discrete-continuous-time formulation of Merton's model with fixed transaction cost, and Syahril (2003b) derives investment strategies for a given transaction intervals. This paper investigates an optimal transaction intervals for portfolio selection problem with fixed transaction cost.

\section{Formulation of the Model}

Formulation of the problem as in Syahril (2003a, 2003b) 


\subsection{Securities Market and Transactions}

It is assumed that a complete probability space $(\Omega, \mathcal{F}, P)$ is given. In addition, it is assumed that a filtration $\left\{\mathcal{F}_{t}: t \geq 0\right\}$ is also given. By a filtration is meant a family of $\sigma$-algebras $\left\{\mathcal{F}_{t}: t \geq 0\right\}$ which is increasing : $\mathcal{F}_{s} \subset \mathcal{F}_{t}$ if $s \leq t$. It is assumed that the one-dimensional standard Brownian motion $B=\left\{B_{t}: t \geq 0\right\}$ is given on a given filtered probability space $\left(\Omega, \mathcal{F},\left(\mathcal{F}_{t}\right)_{0 \leq t<\infty}, P\right)$.

There are two securities available in the economy to an investor. One is a riskless security with fixed interest rate $r$, and the other is a risky security whose price is a geometric Brownian motion with expected rate of return $\alpha$ and rate of return variation $\sigma^{2}$. At time $t \geq 0$, the price processes $\left\{P_{0}(t)\right\}$ of the riskless security satisfy a deterministic differential equation

$$
d P_{0}(t)=r P_{0}(t) d t
$$

while the price processes $\left\{P_{1}(t)\right\}$ of the risky security satisfy a stochastic differential equation

$$
d P_{1}(t)=\alpha P_{1}(t) d t+\sigma P_{1}(t) d B_{t}
$$

Money is available for the investor in the economy as a medium of exchange and numeraire. Only money is exchangable for consumption. Let $M_{t}$ denotes money holdings at time $t$. The investor is assumed to receive no further income from noncapital sources, and starts with the initial stock of money $M_{0}=0$. Trading opportunities are available continuously in time, but with costs. Transaction costs are incurred when information is processed and a portfolio transaction is made. There are two forms of transaction costs: portfolio management fees and withdrawal costs. The investor pays a fraction $\varepsilon>0$ of the total wealth in the securities at the beginning of each interval as a portfolio management fee. The portfolio management fee is meant to include the cost of adjusting the portfolio and the cost of processing information. In this paper, transaction costs is the costs which incurs during withdrawing wealth from the portfolio. The transaction costs is a fixed for every transaction, regardless of amount of wealth transacted. Then the total transaction costs function is of the form $b+\varepsilon\left(X_{\tau_{n}}-W_{\tau_{n}}\right)$, where $X_{\tau_{n}}$ is the total wealth at time $\tau_{n}$ before transaction. Filtration $\left(\mathcal{F}_{t}\right)$ defined by $\mathcal{F}_{t}=\sigma\left\{B_{s}: s \leq t\right\}$, will be interpreted as information available up to time $t$. Given the structure of transaction costs, consumption and investment decisions are made at intervals. During each interval there is no transaction. All dividends of risky security are re-invested continually in the risky security, and all interest income is re-invested continually in the riskless security. 
The investor chooses instants of time at which to process information and make consumption and investment decisions. In other words, information is available continuously through the filtration $\left\{\mathcal{F}_{t}: t \geq 0\right\}$. The investor receives information via controllable filtration

$$
\mathbf{H}=\left\{\mathcal{H}_{t}: t \geq 0\right\} \text { with } \mathcal{H}_{t}=\mathcal{F}_{t}, t \in\left[\tau_{n}, \tau_{n+1}\right),
$$

where $\tau_{n}$ is a $\mathcal{H}_{\tau_{n-1}}$-measurable stopping time at which the $\mathrm{n}$-th transaction occurs. The filtration $\mathbf{H}$ is controllable in the sense that the investor is allowed to choose any sequence $\tau=\left\{\tau_{n}: n=1,2,3, \ldots\right\}$ of such transaction times with $\tau_{1} \equiv 0$. Let $T=\left\{T_{n}=\tau_{n+1}-\tau_{n}: n=1,2,3, \ldots\right\}$ denotes the corresponding sequence of transaction intervals. Finding an optimal stopping policy $\tau$ is clearly equivalent to finding an optimal transaction interval policy $T$.

\section{$2.2 \quad$ Formulation of The Model}

Let the consumption space $\mathcal{C}$ for the investor consists of positive $\mathbf{H}$-adapted consumption processes $\mathbf{C}=\left\{C_{t}: t \geq 0\right\}$ satisfying $\int_{0}^{t} C_{s} d s<\infty$ almost surely for all $t \geq 0$, and

$$
E\left[\int_{0}^{\infty} e^{-\delta t} u\left(C_{t}\right) d t\right]<\infty
$$

where $E$ denotes the expected value function, with respect to $P, \delta$ is a strictly positive scalar discount factor and the utility function $u$, is one of the HARA (hyperbolic absolute risk-aversion) type function, as defined in Merton (1971). We take $u$ as given by

$$
u(C)=\frac{1}{\gamma} C^{\gamma}, \quad 0<\gamma<1
$$

Let $\tau=\left\{\tau_{n}: n=1,2,3, \ldots\right\}$ be sequence of transaction times with $\tau_{1} \equiv 0$. Let $T=\left\{T_{n}=\tau_{n+1}-\tau_{n}, n=1,2,3, \ldots\right\}$ be the sequence of corresponding transaction intervals. Let $W=\left\{W_{\tau_{n}}: n=1,2,3, \ldots\right\}$ be the sequence of money withdrawal processes, and $V=\left\{V_{\tau_{n}}: n=1,2,3, \ldots\right\}$ be the sequence of investment for the risky security.

Let $\mathcal{T}$ denote the space of sequences of strictly positive transaction intervals, $\mathcal{W}$ the space of positive $\mathbf{H}$-adapted money withdrawal processes, and $\mathcal{V}$ the space of $\mathbf{H}$-adapted investment processes for the risky security. Let $\mathcal{U}=$ $\mathcal{T} \times \mathcal{W} \times \mathcal{V} \times \mathcal{C}$

Definition 2.1 A budget policy is a quadruplet $(T, W, V, C) \in \mathcal{U}$. 
We characterize budget feasible policies as follows. Let $\mathcal{U}$ denotes a class of budget policies. Given a policy $(T, W, V, C) \in \mathcal{U}$, then the money holding at any time $t$ is defined by

$$
M_{t}=\sum_{\left\{n: \tau_{n} \leq t\right\}}\left[W_{\tau_{n}}-b\right]-\int_{0}^{t} C_{s} d s,
$$

Let $X_{\tau_{n}}$ denotes the total wealth invested in the securities at time $\tau_{n}$, before the $n$th transaction. Let $W_{\tau_{n}}$ denotes the amount of money withdrawn at time $\tau_{n}$ from the total wealth $X_{\tau_{n}}$, and $V_{\tau_{n}}$ denotes the market value of the investment in the risky security chosen at time $\tau_{n}$. After an amount $W_{\tau_{n}}$ is withdrawn from the total wealth $X_{\tau_{n}}$, and a fraction $\varepsilon$ of the remainder, is paid as management fees, then the wealth left for re-investment is $Z_{\tau_{n}}=(1-\varepsilon)\left[X_{\tau_{n}}-W_{\tau_{n}}\right]$. Of this amount, $V_{\tau_{n}}$ is invested in the risky security with a per-dollar payback of $\Gamma_{n+1}$ at the next transaction date, including continually re-invested dividends. And the remainder, $Z_{\tau_{n}}-V_{\tau_{n}}$, is invested in the riskless security at the continuously compounding interest rate $r>0$.

The investor's total wealth invested at the time of the $(n+1)$ th transaction is therefore

$$
X_{\tau_{n+1}}=(1-\varepsilon)\left[X_{\tau_{n}}-W_{\tau_{n}}\right] e^{r T_{n}}+V_{\tau_{n}}\left[\Gamma_{n+1}-e^{r T_{n}}\right] .
$$

for $n=1,2,3, \ldots$

According to the equation (2) and the Itô's formula, ${ }^{1}$ the return of the risky investment $\Gamma$ satisfies

$$
\Gamma_{n+1}=\exp \left[\left(\alpha-\frac{1}{2} \sigma^{2}\right) T_{n}+\sigma\left(B_{\tau_{n+1}}-B_{\tau_{n}}\right)\right] .
$$

Since $M_{0}=0$, then $X_{0}$ is considered as the initial wealth endowment for the investor.

Definition 2.2 The budget policy $(T, W, V, C) \in \mathcal{U}$ is budget feasible policy if the associated money process $M$ of (5) and invested wealth process $X$ of (6) are non-negative.

\subsection{Optimal Control Statement of the Problem}

Definition 2.3 Let $\mathcal{U}$ be the set of all budget feasible policies as defined previously. The optimal control problem for the investor is to maximize

$$
U\left(X_{0}\right) \equiv \max _{(T, W, V, C) \in \mathcal{U}} E\left[\int_{0}^{\infty} e^{-\delta t} u\left(C_{t}\right) d t\right],
$$

\footnotetext{
${ }^{1}$ Details may be found in Karatzas and Shreve (1988), or Protter (1990)
} 
subject to, for $n=1,2,3, \ldots$,

$$
X_{\tau_{n+1}}=(1-\varepsilon)\left[X_{\tau_{n}}-W_{\tau_{n}}\right] e^{r T_{n}}+V_{\tau_{n}}\left[\Gamma_{n+1}-e^{r T_{n}}\right],
$$

with $M_{t} \geq 0$, and $X_{\tau_{n+1}} \geq 0$.

We assume that only money is available to the investor as a medium of exchange and numeraire in the economy. Only money is exchangeable for consumption. It is also assumed that money cannot be borrowed, it can only be acquired by selling the securities, and it is put in the purse $M$. Because there exists a riskless security with a positive interest rate in the economy, there is no investment demand for money. Duffie and Sun (1990) argued that it will not be optimal for the investor to withdraw more money than the amount needed for financing consumption before the next transaction.

The following result is similar to those in Duffie and Sun (1990), the proof can be found in Duffie and Sun (1990) or in Syahril (2003).

Theorem 2.4 Let the value function $U$ be defined as in (8), and the transaction costs function $\Psi\left(W_{\tau_{n}}\right)=b, \quad b \geq 0$. Then the optimal policy $(T, W, V, C)$ must satisfy for all $n=1,2,3, \ldots$

$$
\int_{\tau_{n}}^{\tau_{n+1}} C_{t} d t=W_{\tau_{n}}-b .
$$

Corollary 1 By the definition of money holding $M_{t}$ of equation (5), then

$$
M_{\tau_{n}}=W_{\tau_{n}}-b, \quad n=1,2,3, \ldots
$$

Therefore, the optimal control problem (8)-(9) is equivalent to the optimal control problem :

$$
U\left(X_{0}\right)=\max _{(T, W, V, C) \in \mathcal{U}} E\left[\int_{0}^{\infty} e^{-\delta t} u\left(C_{t}\right) d t\right]
$$

subject to

$$
\int_{\tau_{n}}^{\tau_{n+1}} C_{t} d t=W_{\tau_{n}}-b
$$

$$
X_{\tau_{n+1}}=(1-\varepsilon)\left[X_{\tau_{n}}-W_{\tau_{n}}\right] e^{r T_{n}}+V_{\tau_{n}}\left[\Gamma_{n+1}-e^{r T_{n}}\right] \geq 0,
$$

for $n=1,2,3, \ldots$.

We summarize the problem faced by the investor in the following definition. For the complete formulation, one can consult ${ }^{2}$.

\footnotetext{
${ }^{2}$ Syahril (2003a)
} 
Definition 2.5 Let $\mathcal{U}$ be the set of all budget feasible policies as defined previously. The optimal control problem for the investor is to maximize

$$
U\left(X_{\tau_{n}}\right)=\max _{\left\{T_{n}, W_{\tau_{n}}, V_{\tau_{n}}\right\}}\left\{Q_{n}^{\nu} \frac{1}{\gamma}\left(W_{\tau_{n}}-b\right)^{\gamma}+e^{-\delta T_{n}} E\left[U\left(X_{\tau_{n+1}}\right) \mid \mathcal{H}_{\tau_{n}}\right]\right\},
$$

subject to

$$
X_{\tau_{n+1}}=(1-\varepsilon)\left[X_{\tau_{n}}-W_{\tau_{n}}\right] e^{r T_{n}}+V_{\tau_{n}}\left[\Gamma_{n+1}-e^{r T_{n}}\right],
$$

for $n=1,2,3, \ldots$, with $M_{t} \geq 0$, and $X_{\tau_{n+1}} \geq 0$.

The following result about optimal investment strategies for the investor was derived in Syahril (2003b), but is presented here for completeness.

Theorem 2.6 Let $T_{n}$ be fixed for $n=1,2,3, \ldots$. Then the optimal value function and unique solution to problem (14)-(15), is given by

$$
U\left(X_{\tau_{n}}\right)=Q_{n}^{\nu} A_{n}^{-\nu} \frac{1}{\gamma}\left(X_{\tau_{n}}-Y_{n}\right)^{\gamma}
$$

with the optimal withdrawal and investment strategies are given by

$$
\begin{aligned}
W_{\tau_{n}} & =A_{n}\left(X_{\tau_{n}}-Y_{n}\right)+b \\
V_{\tau_{n}} & =(1-\varepsilon)\left(1-A_{n}\right)\left(X_{\tau_{n}}-Y_{n}\right) \pi_{n},
\end{aligned}
$$

respectively, and where $A_{n}$, and $Y_{n}$ are given by

$$
\begin{aligned}
A_{n} & =\frac{A_{n+1} Q_{n}}{A_{n+1} Q_{n}+Q_{n+1} R_{n}^{1 / \nu}} \\
Y_{n} & =b+(1-\varepsilon)^{-1} e^{-r T_{n}} Y_{n+1}
\end{aligned}
$$

respectively, with $R_{n}=(1-\varepsilon)^{\gamma} e^{-\delta T_{n}} \Omega_{n}$, and where $\Omega_{n}$ and $\pi_{n}$ are defined by the optimization problem

$$
\begin{aligned}
\Omega_{n} & \equiv E\left(\left[e^{r T_{n}}+\pi_{n}\left(\Gamma_{n+1}-e^{r T_{n}}\right)\right]^{\gamma}\right) \\
& \equiv \sup _{\{0 \leq \pi \leq 1\}} E\left(\left[e^{r T_{n}}+\pi\left(\Gamma_{n+1}-e^{r T_{n}}\right)\right]^{\gamma}\right) .
\end{aligned}
$$

Remark 2.7 If at any $\tau_{n}, W_{\tau_{n}}=b$, then the implications are as follows:

1. $Y_{n}=X_{\tau_{n}}$, by $(17)$.

2. $T_{n}$ can be computed from (20) as

$$
0<T_{n}=\frac{1}{r} \ln \left[\frac{X_{\tau_{n+1}}}{\left(X_{\tau_{n}}-b\right)(1-\varepsilon)}\right]<\infty .
$$


3. $U\left(X_{\tau_{n}}\right)=0$, implying by (8) that $C_{t}=0$, almost every where for $t \geq \tau_{n}$. This can occur only for utility function which satisfy $u(0)=0$.

4. Case 2 and 3 imply that $W_{\tau_{n}}>b$ always in the case of infinite-time horizon

Corollary 2 It is possible that $W_{\tau_{n}}=b$, in which case

$$
T_{n}=\frac{1}{r} \ln \left[\frac{X_{\tau_{n+1}}}{\left(X_{\tau_{n}}-b\right)(1-\varepsilon)}\right] .
$$

This implies that $b<W_{\tau_{n}}$ if the specified $T_{n}$ are not given by (22).

\section{Optimal Transaction Intervals}

Theorem 3.1 Let the problem faced by an investor satisfy relation (14) subject to (15). Then the optimal transaction intervals $T_{n}, n=1,2,3, \ldots$ satisfy

$$
g\left(T_{n}\right)+\left(X_{\tau_{n}}-Y_{n}\right) h\left(T_{n}\right)=0,
$$

where $g$ and $h$ are defined by

$$
g\left(T_{n}\right)=\gamma r(1-\varepsilon)^{-1} e^{-r T_{n}} Y_{n+1}\left[A_{n+1} Q_{n}+Q_{n+1} R_{n}^{1 / \nu}\right],
$$

$$
\begin{aligned}
h\left(T_{n}\right) & =\delta e^{-\frac{\delta}{\nu} T_{n}} A_{n+1}+Q_{n+1} R_{n}^{1 / \nu}\left[-\delta+\gamma r+\gamma(\alpha-r) \pi_{n}\right. \\
& \left.-\frac{1}{2} \sigma^{2} \gamma(1-\gamma) \pi_{n}^{2}\right] .
\end{aligned}
$$

\section{Proof :}

Consider $U$ as in equation (14) which is given by

$$
U\left(X_{\tau_{n}}\right)=\max _{\left\{T_{n}, W_{\tau_{n}}, V_{\tau_{n}}\right\}}\left\{Q_{n}^{\nu} \frac{1}{\gamma}\left[W_{\tau_{n}}-b\right]^{\gamma}+e^{-\delta T_{n}} E\left[U\left(X_{\tau_{n+1}}\right) \mid \mathcal{H}_{\tau_{n}}\right]\right\} .
$$

By differentiation of function $U$ with respect to $W_{\tau_{n}}, V_{\tau_{n}}$ and $T_{n}$ respectively, and setting each of them equals to zero, then necessary conditions for ( 14) for all $n=1,2,3, \ldots$ are :

$$
Q_{n}^{\nu}\left[W_{\tau_{n}}-b\right]^{-\nu}=(1-\varepsilon) e^{-(\delta-r) T_{n}} E\left[U^{\prime}\left(X_{\tau_{n+1}}\right) \mid \mathcal{H}_{\tau_{n}}\right],
$$

$$
E\left[\left(\Gamma_{n+1}-e^{r T_{n}}\right) U^{\prime}\left(X_{\tau_{n+1}}\right) \mid \mathcal{H}_{\tau_{n}}\right]=0
$$




$$
\begin{aligned}
\delta e^{-\frac{\delta}{\nu} T_{n}} Q_{n}^{-\gamma} \frac{1}{\gamma}\left[W_{\tau_{n}}-b\right]^{\gamma}= \\
e^{-\delta T_{n}}\left\{\delta E\left[U\left(X_{\tau_{n+1}}\right) \mid \mathcal{H}_{\tau_{n}}\right]-\frac{\partial E\left[U\left(X_{\tau_{n+1}}\right) \mid \mathcal{H}_{\tau_{n}}\right]}{\partial T_{n}}\right\} .
\end{aligned}
$$

211

Apparently processes $\left\{Z_{t}\right\}$ is a martingale. ${ }^{4}$ Therefore,

$$
\begin{aligned}
\frac{\partial E\left[U\left(X_{\tau_{n+1}}\right) \mid \mathcal{H}_{\tau_{n}}\right]}{\partial T_{n}} & =E\left[\left[r\left(X_{\tau_{n+1}}-G_{\tau_{n+1}}\right)+\alpha G_{\tau_{n+1}}\right] U^{\prime}\left(X_{\tau_{n+1}}\right)\right. \\
& \left.+\frac{1}{2} \sigma^{2} G_{\tau_{n+1}}^{2} U^{\prime \prime}\left(X_{\tau_{n+1}}\right) \mid \mathcal{H}_{\tau_{n}}\right] .
\end{aligned}
$$

\footnotetext{
${ }^{3}$ See for example Theorem 32 of Protter (1990)

${ }^{4}$ For details, see appendix 3 of Duffie and Sun (1990)
} 
Substitution of relation (28) into (26) results in

$$
\begin{aligned}
& \delta e^{-\frac{\delta}{\nu} T_{n}} Q_{n}^{-\gamma} \frac{1}{\gamma}\left[W_{\tau_{n}}-b\right]^{\gamma}= \\
& \delta e^{-\delta T_{n}} E\left[U\left(X_{\tau_{n+1}}\right) \mid \mathcal{H}_{\tau_{n}}\right]-e^{-\frac{\delta}{\nu} T_{n}} E\left[\left[r\left(X_{\tau_{n+1}}-G_{\tau_{n}+1}\right)\right.\right. \\
& \left.\left.\quad+\alpha G_{\tau_{n+1}}\right] U^{\prime}\left(X_{\tau_{n+1}}\right)+\frac{1}{2} \sigma^{2} G_{\tau_{n+1}}^{2} U^{\prime \prime}\left(X_{\tau_{n+1}}\right) \mid \mathcal{H}_{\tau_{n}}\right] .
\end{aligned}
$$

Remark 3.2 Duffie and Sun (1990) have used $V_{\tau_{n+1}}$ in place of $G_{\tau_{n+1}}$.

Notice that by insertion of (17) and (18) into (15), then the total wealth process $X_{\tau_{n+1}}$ may be re-written as

$$
X_{\tau_{n+1}}=(1-\varepsilon)\left(1-A_{n}\right)\left(X_{\tau_{n}}-Y_{n}\right)\left[e^{r T_{n}}+\pi_{n}\left(\Gamma_{n+1}-e^{r T_{n}}\right)\right]+Y_{n+1} .
$$

Since $G_{\tau_{n+1}}=V_{\tau_{n}} \Gamma_{n+1}$, then by application of (18) we also have

$$
G_{\tau_{n+1}}=(1-\varepsilon)\left(1-A_{n}\right)\left(X_{\tau_{n}}-Y_{n}\right) \pi_{n} \Gamma_{n+1} .
$$

Applying (30) in (16) then we have the following equations :

$$
\begin{aligned}
E\left[U\left(X_{\tau_{n+1}}\right) \mid \mathcal{H}_{\tau_{n}}\right] & =(1-\varepsilon)^{\gamma}\left(1-A_{n}\right)^{\gamma} \frac{1}{\gamma}\left(X_{\tau_{n}}-Y_{n}\right)^{\gamma} \\
& \times E\left(\left[e^{r T_{n}}+\pi_{n}\left(\Gamma_{n+1}-e^{r T_{n}}\right)\right]^{\gamma}\right) Q_{n+1}^{\nu} A_{n+1}^{-\nu} \\
E\left[U^{\prime}\left(X_{\tau_{n+1}}\right) \mid \mathcal{H}_{\tau_{n}}\right] & =(1-\varepsilon)^{-\nu}\left(1-A_{n}\right)^{-\nu}\left(X_{\tau_{n}}-Y_{n}\right)^{-\nu} \\
& \times E\left(\left[e^{r T_{n}}+\pi_{n}\left(\Gamma_{n+1}-e^{r T_{n}}\right)\right]^{-\nu}\right) Q_{n+1}^{\nu} A_{n+1}^{-\nu} \\
E\left[U^{\prime \prime}\left(X_{\tau_{n+1}}\right) \mid \mathcal{H}_{\tau_{n}}\right] & =(1-\varepsilon)^{-\nu-1}\left(1-A_{n}\right)^{-\nu-1}\left(X_{\tau_{n}}-Y_{n}\right)^{-\nu-1} \\
& \times(-\nu) E\left(\left[e^{r T_{n}}+\pi_{n}\left(\Gamma_{n+1}-e^{r T_{n}}\right)\right]^{-\nu-1}\right) Q_{n+1}^{\nu} A_{n+1}^{-\nu} .
\end{aligned}
$$

From the definition of $\Omega_{n}$ in (21), then its derivative with respect to $\pi_{n}$ gives

$$
E\left(\left[e^{r T_{n}}+\pi_{n}\left(\Gamma_{n+1}-e^{r T_{n}}\right)\right]^{-\nu}\left[\Gamma_{n+1}-e^{r T_{n}}\right]\right)=0 .
$$

This implies that the following relations hold :

$$
\begin{aligned}
\Omega_{n} & =E\left(e^{r T_{n}}\left[e^{r T_{n}}+\pi_{n}\left(\Gamma_{n+1}-e^{r T_{n}}\right)\right]^{-\nu}\right), \\
& =E\left(\Gamma_{n+1}\left[e^{r T_{n}}+\pi_{n}\left(\Gamma_{n+1}-e^{r T_{n}}\right)\right]^{-\nu}\right), \\
& =E\left(\Gamma_{n+1}^{2}\left[e^{r T_{n}}+\pi_{n}\left(\Gamma_{n+1}-e^{r T_{n}}\right)\right]^{-\nu-1}\right) .
\end{aligned}
$$

By substituting previous equations and (17), (31), (35), (36), (37) into (29), and by dividing all terms by $(1 / \gamma)\left(X_{\tau_{n}}-Y_{n}\right)^{\gamma-1}$, and by rearranging the terms, then we have the following equation :

$$
\begin{gathered}
\left(X_{\tau_{n}}-Y_{n}\right)\left\{\delta e^{-\frac{\delta}{\nu} T_{n}} Q_{n}^{-\gamma} A_{n}^{\gamma}+Q_{n+1}^{\nu} A_{n+1}^{-\nu}\left(1-A_{n}\right)^{\gamma} R_{n}[-\delta+\gamma r\right. \\
\left.\left.+\gamma(\alpha-r) \pi_{n}-\frac{1}{2} \sigma^{2} \gamma(1-\gamma) \pi_{n}^{2}\right]\right\} \\
+\gamma r(1-\varepsilon)^{-1} e^{-r T_{n}} Q_{n+1}^{\nu} A_{n+1}^{-\nu}\left(1-A_{n}\right)^{-\nu} R_{n} Y_{n+1}=0 .
\end{gathered}
$$


By applications of $A_{n}$ of (19) and $Y_{n}$ of (20) in (38), then we have

$$
\begin{aligned}
& \left(X_{\tau_{n}}-Y_{n}\right)\left\{\delta e^{-\frac{\delta}{\nu} T_{n}} \frac{A_{n+1}^{\gamma}}{\left[A_{n+1} Q_{n}+Q_{n+1} R_{n}^{1 / \nu}\right] \gamma}+Q_{n+1}^{\nu} A_{n+1}^{-\nu} R_{n}\right. \\
& \left.\times \frac{Q_{n+1}^{\gamma} R_{n}^{\gamma / \nu}}{\left[A_{n+1} Q_{n}+Q_{n+1} R_{n}^{1 / \nu}\right] \gamma}\left[-\delta+\gamma r+\gamma(\alpha-r) \pi_{n}-\frac{1}{2} \sigma^{2} \gamma(1-\gamma) \pi_{n}^{2}\right]\right\} \\
& +\frac{\gamma r Y_{n+1} R_{n}}{(1-\varepsilon) e^{r T_{n}} Q_{n+1}^{\nu} A_{n+1}^{-\nu}} \frac{Q_{n+1}^{-\nu} R_{n}^{-\nu / \nu}}{\left[A_{n+1} Q_{n}+Q_{n+1} R_{n}\right]^{-\nu}}=0
\end{aligned}
$$

Finally, by dividing all terms of (39) by $\frac{A_{n+1}^{-\nu}}{\left[A_{n+1} Q_{n}+Q_{n+1} R_{n}^{1 / \nu}\right]^{\gamma}}$ results in the following relation :

$$
g\left(T_{n}\right)+\left(X_{\tau_{n}}-Y_{n}\right) h\left(T_{n}\right)=0,
$$

where $g$ and $h$ are defined as the following :

$$
g\left(T_{n}\right)=\gamma r(1-\varepsilon)^{-1} e^{-r T_{n}} Y_{n+1}\left[A_{n+1} Q_{n}+Q_{n+1} R_{n}^{1 / \nu}\right]
$$

$$
\begin{aligned}
h\left(T_{n}\right) & =\delta e^{-\frac{\delta}{\nu} T_{n}} A_{n+1}+Q_{n+1} R_{n}^{1 / \nu}\left[-\delta+\gamma r+\gamma(\alpha-r) \pi_{n}\right. \\
& \left.-\frac{1}{2} \sigma^{2} \gamma(1-\gamma) \pi_{n}^{2}\right] .
\end{aligned}
$$

Therefore the proof of the theorem has been completed

Theorem 3.3 Suppose that $\alpha, \delta, \varepsilon, \gamma, r, \sigma$ satisfying the following conditions :

$$
\begin{aligned}
& \text { 1. } \alpha-r \geq \frac{1}{2} \sigma^{2}(1-\gamma) \\
& \text { 2. } \max (\gamma \alpha, \gamma r)<\delta<\min \left(\frac{\gamma r}{(1-\varepsilon)^{\gamma / \nu}}, r-\gamma r\right)
\end{aligned}
$$

If $A_{n}$ as given by (19) has a property such that $A_{n} \geq Q_{n}\left(1-R_{n}^{1 / \nu}\right)$, then the equation

$$
g\left(T_{n}\right)+\left(X_{\tau_{n}}-Y_{n}\right) h\left(T_{n}\right)=0
$$

as defined in Theorem 3.1 has a solution.

Proof : Let $H\left(T_{n}\right)=g\left(T_{n}\right)+\left(X_{\tau_{n}}-Y_{n}\right) h\left(T_{n}\right)$, where $g$ and $h$ are defined by (24) and (25) respectively. Note that $H$ is a continuous function 
256 Therefore,

$$
\lim _{T_{n} \rightarrow 0^{+}} H\left(T_{n}\right)>\gamma r(1-\varepsilon)^{-1+\gamma / \nu} Q_{n+1} Y_{n+1} .
$$

257 relation (20). Therefore, its definition, $0 \leq \pi_{n} \leq 1$. These imply that

$$
-\delta+\gamma r+\gamma(\alpha-r) \pi_{n}-\frac{1}{2} \sigma^{2} \gamma(1-\gamma) \pi_{n}^{2} \geq-\delta+\gamma r
$$

And since $\lim _{T_{n} \rightarrow 0^{+}} R_{n}^{1 / \nu}=(1-\varepsilon)^{\gamma / \nu}$, then we have that

$$
\begin{aligned}
\lim _{T_{n} \rightarrow 0^{+}} H\left(T_{n}\right) & \geq \gamma r(1-\varepsilon)^{-1} Y_{n+1} Q_{n+1}(1-\varepsilon)^{\gamma / \nu} \\
& +Q_{n+1}\left(X_{\tau_{n}}-b-(1-\varepsilon)^{-1} Y_{n+1}\right) \\
& \times\left\{\delta\left[1-(1-\varepsilon)^{\gamma / \nu}\right](1-\varepsilon)^{\gamma / \nu}+(1-\varepsilon)^{\gamma / \nu}[-\delta+\gamma r]\right\} .
\end{aligned}
$$

By the assumption of the Theorem, $\delta<\frac{\gamma r}{(1-\varepsilon)^{\gamma / \nu}}$. This implies that

$$
\delta\left[1-(1-\varepsilon)^{\gamma / \nu}\right](1-\varepsilon)^{\gamma / \nu}+(1-\varepsilon)^{\gamma / \nu}[-\delta+\gamma r]>0 .
$$

Since $\gamma, r, \varepsilon, Y_{n+1}, Q_{n+1}$ are nonnegatives, hence

$$
\begin{aligned}
\lim _{T_{n} \rightarrow 0^{+}} H\left(T_{n}\right) & =\lim _{T_{n} \rightarrow 0^{+}} \gamma r(1-\varepsilon)^{-1} e^{-r T_{n}} Y_{n+1}\left[A_{n+1} Q_{n}+Q_{n+1} R_{n}^{1 / \nu}\right] \\
& +\lim _{T_{n} \rightarrow 0^{+}} Q_{n+1}\left(X_{\tau_{n}}-Y_{n}\right)\left\{\delta e^{-\delta / \nu T_{n}} \frac{A_{n+1}}{Q_{n+1}}\right. \\
& \left.+R_{n}^{1 / \nu}\left[-\delta+\gamma r+\gamma(\alpha-r) \pi_{n}-1 / 2 \sigma^{2} \gamma(1-\gamma) \pi_{n}^{2}\right]\right\} .
\end{aligned}
$$

But $A_{n}$ has the property that either $A_{n} \geq Q_{n}\left(1-R_{n}^{1 / \nu}\right)$, or $A_{n}<$ $Q_{n}\left(1-R_{n}^{1 / \nu}\right)$. Since we have assumed that $A_{n} \geq Q_{n}\left(1-R_{n}^{1 / \nu}\right)$, therefore, $\frac{A_{n+1}}{Q_{n+1}} \geq\left(1-R_{n}^{1 / \nu}\right) R_{n}^{1 / \nu}$. Furthermore, $Y_{n}=b+(1-\varepsilon)^{-1} e^{-r T_{n}} Y_{n+1}$ by

$$
\begin{aligned}
\lim _{T_{n} \rightarrow 0^{+}} H\left(T_{n}\right) & \geq \lim _{T_{n} \rightarrow 0^{+}} \gamma r(1-\varepsilon)^{-1} e^{-r T_{n}} Y_{n+1}\left[A_{n+1} Q_{n}+Q_{n+1} R_{n}^{1 / \nu}\right] \\
& +\lim _{T_{n} \rightarrow 0^{+}} Q_{n+1}\left(X_{\tau_{n}}-b-(1-\varepsilon)^{-1} e^{-r T_{n}} Y_{n+1}\right) \\
& \times\left\{\delta e^{-\delta / \nu T_{n}}\left(1-R_{n}^{1 / \nu}\right) R_{n}^{1 / \nu}+R_{n}^{1 / \nu}[-\delta+\gamma r\right. \\
& \left.\left.+\gamma(\alpha-r) \pi_{n}-1 / 2 \sigma^{2} \gamma(1-\gamma) \pi_{n}^{2}\right]\right\} .
\end{aligned}
$$

By the assumption of the theorem, $(\alpha-r) \geq \frac{1}{2} \sigma^{2}(1-\gamma)$. In addition, by

$$
\lim _{T_{n} \rightarrow 0^{+}} H\left(T_{n}\right)>0 .
$$


On the other hand, since $A_{n+1}, Q_{n+1}, Q_{n}$ are nonnegatives and less than one, and $R_{n} \leq(1-\varepsilon)^{\gamma}$, then

$$
\begin{aligned}
H\left(T_{n}\right) & =g\left(T_{n}\right)+\left(X_{\tau_{n}}-Y_{n}\right) h\left(T_{n}\right) \\
& =\frac{\gamma r}{(1-\varepsilon) e^{r T_{n}}} Y_{n+1}\left[A_{n+1} Q_{n}+Q_{n+1} R_{n}^{1 / \nu}\right] \\
& +\left(X_{\tau_{n}}-Y_{n}\right)\left\{\delta e^{-\frac{\delta}{\nu} T_{n}} A_{n+1}+Q_{n+1} R_{n}^{1 / \nu}\right. \\
& \left.\times\left[-\delta+\gamma r+\gamma(\alpha-r) \pi_{n}-\frac{1}{2} \sigma^{2} \gamma(1-\gamma) \pi_{n}^{2}\right]\right\} \\
& \leq \frac{\gamma r Y_{n+1}\left[1+(1-\varepsilon)^{\gamma / \nu}\right]}{(1-\varepsilon) e^{r T_{n}}} \\
& +\left(X_{\tau_{n}}-Y_{n}\right)\left\{\delta e^{-\frac{\delta}{\nu} T_{n}}+Q_{n+1} R_{n}^{1 / \nu}[-\delta+\max (\gamma \alpha, \gamma r)]\right\}
\end{aligned}
$$

By re-arranging all terms, then

$$
\begin{aligned}
H\left(T_{n}\right) & \leq e^{-\frac{\delta}{\nu} T_{n}}\left(X_{\tau_{n}}-Y_{n}\right)\left\{\frac{\gamma r Y_{n+1} e^{\frac{\delta}{\nu} T_{n}}\left[1+(1-\varepsilon)^{\gamma / \nu}\right]}{\left(X_{\tau_{n}}-Y_{n}\right)(1-\varepsilon) e^{r T_{n}}}\right. \\
& \left.+\delta+e^{\delta / \nu T_{n}} Q_{n+1} R_{n}^{1 / \nu}[-\delta+\max (\gamma \alpha, \gamma r)]\right\}
\end{aligned}
$$

Since, by assumptions of Theorem 3.3 that $\delta>\max (\gamma \alpha, \gamma r)$, and $r>\delta / \nu$, then $H\left(T_{n}\right)<0$ for large $T_{n}$. It has been shown that $H\left(T_{n}\right)>0$ as $T_{n} \rightarrow 0^{+}$, and $H\left(T_{n}\right)<0$ for large $T_{n}$. Since $H\left(T_{n}\right)$ is a continuous function in $T_{n}$, then there exists $\hat{T}_{n}$, such that $H\left(\hat{T}_{n}\right)=0$. Therefore, the proof of Theorem 3.3 has been completed

Theorem 3.4 Suppose that $\alpha, \delta, \varepsilon, \gamma, r, \sigma$ satisfying the following conditions :

$$
\begin{aligned}
& \text { 1. } \alpha-r \geq \frac{1}{2} \sigma^{2}(1-\gamma) \\
& \text { 2. } \max (\gamma \alpha, \gamma r)<\delta<\min \left(\frac{\gamma r}{(1-\varepsilon)^{\gamma / \nu}}, r-\gamma r\right)
\end{aligned}
$$

If $A_{n}$ as given by (19) has the property such that $A_{n} \geq Q_{n}\left(1-R_{n}^{1 / \nu}\right)$, then an optimal policy $(T, W, V, C)$ exists.

Proof : We have that $\frac{A_{n+1}}{Q_{n+1}} \geq\left(1-R_{n}^{1 / \nu}\right) R_{n}^{1 / \nu}$ for $A_{n} \geq Q_{n}\left(1-R_{n}^{1 / \nu}\right)$. By Theorem 3.3, then there exists a scalar $\hat{T}_{n}>0$ such that $H\left(\hat{T}_{n}\right)=0$. The arguments in the proof of Theorem 3.3 show that one of the solutions, say $\hat{T}_{n}$, corresponds to the maximum of (14). 
Now consider the following withdrawal and investment policy and function $f$, for $n=1,2,3, \ldots$

$$
\begin{aligned}
f\left(X_{\tau_{n}}\right) & =Q_{n}^{\nu} A_{n}^{-\nu} \frac{1}{\gamma}\left(X_{\tau_{n}}-Y_{n}\right)^{\gamma}, \\
W_{\tau_{n}} & =A_{n}\left(X_{\tau_{n}}-Y_{n}\right)+b, \\
V_{\tau_{n}} & =(1-\varepsilon)\left(1-A_{n}\right)\left(X_{\tau_{n}}-Y_{n}\right) \pi_{n}, \\
T_{n} & =\hat{T}_{n} .
\end{aligned}
$$

From the preceeding calculations, we know that $f\left(X_{\tau_{n}}\right)$ satisfies ( 14) $\forall n$. It will be shown that $\lim _{n \rightarrow \infty} E\left[e^{-\delta \tau_{n}} f\left(X_{\tau_{n}}\right)\right]=0$. By using (43), then

$$
E\left[e^{-\delta \tau_{n+1}} f\left(X_{\tau_{n+1}}\right) \mid \mathcal{H}_{\tau_{n}}\right]=Q_{n+1}^{\nu} A_{n+1}^{-\nu} e^{-\delta \tau_{n+1}} \frac{1}{\gamma} E\left[\left(X_{\tau_{n+1}}-Y_{n+1}\right)^{\gamma} \mid \mathcal{H}_{\tau_{n}}\right],
$$

with the total wealth $X_{\tau_{n+1}}$ is given by (30).

By substitution of (44) and (45) into the total wealth $X_{\tau_{n+1}}$ of (30), then the expectation factor on the right hand side of (47) may be written as

$$
E\left[\left(X_{\tau_{n+1}}-Y_{n+1}\right)^{\gamma} \mid \mathcal{H}_{\tau_{n}}\right]=(1-\varepsilon)^{\gamma} \Omega_{n}\left(1-A_{n}\right)^{\gamma}\left[\left(X_{\tau_{n}}-Y_{n}\right)^{\gamma} \mid \mathcal{H}_{\tau_{n-1}}\right] .
$$

Since $R_{n}=(1-\varepsilon)^{\gamma} e^{-\delta T_{n}} \Omega_{n}$, and $\tau_{n+1}=T_{n}+\tau_{n}$, then the right hand side of (47) may be written as

$$
\begin{aligned}
& \frac{1}{\gamma} Q_{n+1}^{\nu} A_{n+1}^{-\nu} e^{-\delta \tau_{n+1}} E\left[\left(X_{\tau_{n+1}}-Y_{n+1}\right)^{\gamma} \mid \mathcal{H}_{\tau_{n}}\right] \\
& =\frac{1}{\gamma} Q_{n+1}^{\nu} A_{n+1}^{-\nu} e^{-\delta T_{n}}(1-\varepsilon)^{\gamma} \Omega_{n}\left(1-A_{n}\right)^{\gamma} e^{-\delta \tau_{n}}\left[\left(X_{\tau_{n}}-Y_{n}\right)^{\gamma} \mid \mathcal{H}_{\tau_{n-1}}\right] \\
& =\frac{1}{\gamma} Q_{n+1}^{\nu} A_{n+1}^{-\nu} R_{n}\left(1-A_{n}\right)^{\gamma} e^{-\delta \tau_{n}}\left[\left(X_{\tau_{n}}-Y_{n}\right)^{\gamma} \mid \mathcal{H}_{\tau_{n-1}}\right] .
\end{aligned}
$$

286 Hence, we have

$$
E\left[e^{-\delta \tau_{n+1}} f\left(X_{\tau_{n+1}}\right) \mid \mathcal{H}_{\tau_{n}}\right]=\frac{1}{\gamma} Q_{n+1}^{\nu} A_{n+1}^{-\nu} R_{n}\left(1-A_{n}\right)^{\gamma} e^{-\delta \tau_{n}}\left[\left(X_{\tau_{n}}-Y_{n}\right)^{\gamma} \mid \mathcal{H}_{\tau_{n-1}}\right] .
$$

Let $D=\frac{1}{\gamma} Q_{n+1}^{\nu} A_{n+1}^{-\nu}$.

Then by induction we have the following :

$$
\begin{aligned}
E & {\left[e^{-\delta \tau_{n+1}} f\left(X_{\tau_{n+1}}\right)\right] } \\
& =D R_{n}\left(1-A_{n}\right)^{\gamma} e^{-\delta \tau_{n}}\left[\left(X_{\tau_{n}}-Y_{n}\right)^{\gamma} \mid \mathcal{H}_{\tau_{n-1}}\right] \\
& =D R_{n}\left(1-A_{n}\right)^{\gamma} R_{n-1}\left(1-A_{n-1}\right)^{\gamma} e^{-\delta \tau_{n-1}}\left[\left(X_{\tau_{n-1}}-Y_{n-1}\right)^{\gamma} \mid \mathcal{H}_{\tau_{n-2}}\right] \\
& =D R_{n}\left(1-A_{n}\right)^{\gamma} \ldots R_{1}\left(1-F_{1}\right)^{\gamma} e^{-\delta \tau_{1}}\left[\left(X_{\tau_{1}}-Y_{1}\right)^{\gamma} \mid \mathcal{H}_{\tau_{1}}\right] \\
& \leq D X_{0}^{\gamma} \prod_{k=1}^{n}\left[R_{k}\left(1-A_{k}\right)^{\gamma}\right] \leq D X_{0}^{\gamma} \prod_{k=1}^{n}\left[(1-\varepsilon)^{\gamma / \nu}\right],
\end{aligned}
$$


as $0<1-A_{n}<1$, and $R_{n} \leq(1-\varepsilon)^{\gamma / \nu} \forall n$. Since $D$ is bounded, then $\lim _{n \rightarrow \infty} E\left[e^{-\delta \tau_{n}} f\left(X_{\tau_{n}}\right)\right]=0$.

Therefore, the proof of Theorem 3.4 has been completed

\section{Equal Intervals}

Now consider the case of transaction costs $b=0$. Let transaction intervals $T_{1}=T_{2}=\ldots=K$. This implies that $\tau_{n}=(n-1) K$. With $T_{n}$ are equals for all $\mathrm{n}$, then $A_{n+1}=A_{n}, \quad Q_{n+1}=Q_{n}, \quad Y_{n+1}=Y_{n}$. By replacing $A_{n+1}$ with $A_{n}$ and $Q_{n+1}$ with $Q_{n}$ in (19), and replacing $Y_{n+1}$ with $Y_{n}$ in (20), respectively, result in

$$
A_{n}=1-\left[(1-\varepsilon)^{\gamma} e^{-\delta T_{n}} \Omega_{n}\right]^{1 / \nu},
$$

and

$$
Y_{n}=\frac{(1-\varepsilon) e^{r T_{n}} b}{(1-\varepsilon) e^{r T_{n}}-1}
$$

Note that transaction costs function $b=0$ implies $Y_{n}=0$. This implies that $g\left(T_{n}\right)+\left(X_{\tau_{n}}-Y_{n}\right) h\left(T_{n}\right)=0$ is equivalent to $h\left(T_{n}\right)=0$, where $h$ is given by

$$
\begin{aligned}
h\left(T_{n}\right) & =\delta e^{-\frac{\delta}{\nu} T_{n}} A_{n}+Q_{n} R_{n}^{1 / \nu}\left[-\delta+\gamma r+\gamma(\alpha-r) \pi_{n}\right. \\
& \left.-\frac{1}{2} \sigma^{2} \gamma \nu \pi_{n}^{2}\right] .
\end{aligned}
$$

The following result is proved in Duffie and $\operatorname{Sun}(1990)$, however the proof is incomplete.

Theorem 4.1 Suppose that $\delta>\max (\gamma \alpha, \gamma r)$, and that $b=0$. Then $h\left(T_{n}\right)=0$ has a solution.

Proof : From its definition, $h$ is a continuous function in $T_{n}$. It will be shown that $h>0$, as $T_{n}$ approaches zero, and $h<0$, as $T_{n}$ goes toward infinity. Since $Q_{n}=1-e^{-\frac{\delta}{\nu} T_{n}}$, then

$$
\lim _{T_{n} \rightarrow 0^{+}} h\left(T_{n}\right)=\lim _{T_{n} \rightarrow 0^{+}} \delta e^{-\frac{\delta}{\nu} T_{n}} A_{n} .
$$

Note that

$\lim _{T_{n} \rightarrow 0^{+}} \exp \left[(-\delta+\gamma r) T_{n}\right]=1, \quad$ and $\lim _{T_{n} \rightarrow 0^{+}} \exp \left[(-\delta+\max (\gamma \alpha, \gamma r)) T_{n}\right]=1$.

310 Since

$$
e^{(-\delta+\gamma r) T_{n}} \leq e^{-\delta T_{n}} \Omega_{n} \leq e^{[-\delta+\max (\gamma \alpha, \gamma r)] T_{n}},
$$


then $\lim _{T_{n} \rightarrow 0^{+}} e^{-\delta T_{n}} \Omega_{n}=1$. Therefore,

$$
\lim _{T_{n} \rightarrow 0^{+}} h\left(T_{n}\right)=\delta\left[1-(1-\varepsilon)^{\gamma / \nu}\right]>0 .
$$

On the other hand,

$$
\begin{aligned}
h\left(T_{n}\right) & \leq \delta e^{-\frac{\delta}{\nu} T_{n}} A_{n}+\left(1-A_{n}\right) Q_{n} \\
& \times\left[\gamma r+\gamma(\alpha-r) \pi_{n}-\delta\right] \\
& \leq e^{-\frac{\delta}{\nu} T_{n}}\left[\delta+(\max (\gamma \alpha, \gamma r)-\delta)(1-\varepsilon)^{\gamma / \nu} e^{\frac{\gamma r}{\nu} T_{n}}\right] .
\end{aligned}
$$

Since by the assumption of Theorem 4.1 that $\delta>\max (\gamma \alpha, \gamma r)$, then for large $T_{n}, \quad h\left(T_{n}\right)<0$. Since $h$ is continuous in $T_{n}$, therefore there exists $\bar{T}_{n}$ such that $h\left(\bar{T}_{n}\right)=0$. Hence, the proof of Theorem 4.1 has been completed

Theorem 4.2 Suppose that $\delta>\max (\gamma \alpha, \gamma r)$ and that $b=0$. Then an optimal policy $(T, W, V, C)$ exists and the optimal transaction intervals $\left\{T_{n}\right\}$ are equal.

Proof : The proof is as in Duffie and Sun(1990). With $b=0, H\left(T_{n}\right)=0$ is equivalent to $h\left(T_{n}\right)=0$, for all $\mathrm{n}$. By Theorem 4.1, $h\left(T_{n}\right)=0$, has a solution. Therefore, there exists $\bar{T}_{n}$ such that $h\left(\bar{T}_{n}\right)=0$.

Now consider the following withdrawal and investment policy and function $f$, for $\mathrm{n}=1,2,3, \ldots$

$$
\begin{aligned}
f\left(X_{\tau_{n}}\right) & =Q_{n}^{\nu} A_{n}^{-\nu} \frac{1}{\gamma} X_{\tau_{n}}^{\gamma}, \\
W_{\tau_{n}} & =A_{n} X_{\tau_{n}}, \\
V_{\tau_{n}} & =(1-\varepsilon)\left(1-A_{n}\right) X_{\tau_{n}} \pi_{n}, \\
T_{n} & =\bar{K} .
\end{aligned}
$$

From the preceeding calculations, we know that that $f\left(X_{\tau_{n}}\right)$ satisfies (??) and that $\left(T_{n}, W_{\tau_{n}}, V_{\tau_{n}}\right)$ achieves the maximum in (??) $\forall n$.

Let $D \equiv(1 / \gamma) Q_{n+1}^{\nu} A_{n+1}^{-\nu}$. Therefore,

$$
\begin{aligned}
E & {\left[e^{-\delta \tau_{n+1}} f\left(X_{\tau_{n+1}}\right) \mid \mathcal{H}_{\tau_{n}}\right]=e^{-\delta\left(T_{n}+\tau_{n}\right)} D E\left[X_{\tau_{n+1}}^{\gamma} \mid \mathcal{H}_{\tau_{n}}\right] } \\
& =D e^{-\delta T_{n}}(1-\varepsilon)^{\gamma} \Omega_{n}\left(1-A_{n}\right)^{\gamma} e^{-\delta \tau_{n}}\left[X_{\tau_{n}}^{\gamma} \mid \mathcal{H}_{\tau_{n-1}}\right] \\
& =D R_{n}\left(1-A_{n}\right)^{\gamma} e^{-\delta \tau_{n}}\left[X_{\tau_{n}}^{\gamma} \mid \mathcal{H}_{\tau_{n-1}}\right] \\
& =D R_{n}\left(1-A_{n}\right)^{\gamma}(1-\varepsilon)^{\gamma} \Omega_{n-1}\left(1-A_{n-1}\right)^{\gamma} e^{-\delta \tau_{n-1}}\left[X_{\tau_{n-1}}^{\gamma} \mid \mathcal{H}_{\tau_{n-2}}\right] \\
& =D R_{n}\left(1-A_{n}\right)^{\gamma} R_{n-1}\left(1-A_{n-1}\right)^{\gamma} \ldots R_{1}\left(1-A_{1}\right)^{\gamma} X_{0}^{\gamma} \\
& =D X_{0}^{\gamma} \prod_{k=1}^{n} R_{k}\left(1-A_{k}\right)^{\gamma}=D X_{0}^{\gamma}\left[\left[e^{-\delta \bar{K}}(1-\varepsilon)^{\gamma} \Omega_{n}\right]^{1 / \nu}\right]^{n},
\end{aligned}
$$


as $1-A_{n}=R_{n}^{1 / \nu}=\left[(1-\varepsilon)^{\gamma} \Omega_{n} e^{-\delta \bar{K}}\right], \quad \forall n$. Since $\left[(1-\varepsilon)^{\gamma} \Omega_{n} e^{-\delta \bar{K}}\right]<$ $(1-\varepsilon)^{\gamma}$, for any feasible policy, then $\lim _{n \rightarrow \infty} E\left[e^{-\delta \tau_{n}} f\left(X_{\tau_{n}}\right)\right]=0$. Hence, the proof of Theorem 4.1 has been completed

\section{Conclusion}

We have established an optimal transaction interval for a consumption and investment selection problem for an individual who seeks to maximize the expected utility of consumption. The individual has available a riskless asset with fixed interest rate and a risky one with logarithmic Brownian motion price fluctuations. The individual observes current wealth when making transaction, and decisions to transact can be made at any time, but not without costs. The individual is charged a fixed fraction $\epsilon>0$ of the current wealth as a portfolio management fee plus fixed transaction costs. The problem was formulated in discrete-continuous-time stochastic optimal control problem.

It has been shown that transaction intervals satisfied a nonlinear equation, and it has been shown the existence of the nonlinear equation. The general result is that the optimal transaction intervals $T_{n}$ depend on the amount of total wealth at the beginning of each intervals.

If, for any reason that transactions do not incur costs other than those of management fee, it was shown that the optimal interval between transactions are independent of wealth at the beginning of the interval. This result verifies those of Duffie and Sun (1990). This is so because Duffie and Sun (1990) actually treated linear transaction costs by transforming it to fixed transaction costs problem. Then by taking $b=0$, Duffie and Sun (1990) provide result only for the proportional transaction costs problem.

\section{References}

[1] Constantinides, G.M., 1979, Multiperiod Consumption and Investment Behaviour with Transaction Costs, Management Science, 25(11), pp. 1127-1137

[2] Constantinides, G.M., 1986, Capital Market Equilibrium with Transaction Costs, Journal of Political Economy, 94(4), pp. 842-862

[3] Cox, J.C and C.F Huang, 1989, Optimal Consumption and Portfolio Policies when Asset Prices Follow a Diffusion Process, Journal of Economic Theory, 49, pp. $33-83$

[4] Davis, M.H.A and A.R Norman, 1990, Portfolio Selection with Transaction Costs, Mathematics of Operations Research, 15(4), pp. 676-713 
[5] Duffie, D and T.S Sun, 1990, Transaction Costs and Portfolio Choice in a Discrete-Continuous-Time Setting, Journal of Economic Dynamics and Control, 14, pp. 35-51

[6] Dumas, B. and E. Luciano, 1991, An Exact Solution to a Dynamic Portfolio Choice Problem Under Transaction Costs, Journal of Finance, XLVI(2), pp. 577-595

[7] Karatzas, I. and S.E. Shreve, 1988, Brownian Motion and Stochastic Calculus, Springer-Verlag, New York

[8] Lelands, H.E., 1985, Option Pricing and Replication with Transaction Costs, The Journal of Finance, XL(5), pp. 1283-1301

[9] Magill M.J.P and G.M. Constantinides, 1976, Portfolio Selection with Transaction Costs, Journal of Economic Theory, 13, pp. 245-263

[10] Merton, R.C., 1990, Continuous-Time Finance, Blackwell, Cambridge

[11] Merton, R.C., 1971, Optimum Consumption and Portfolio Rules in Continuous Time Model, Journal of Economic Theory, 3, pp. 373-413

[12] Protter, P., 1990, Stochastic Intergration and Differential Equations, SpringerVerlag, Berlin 